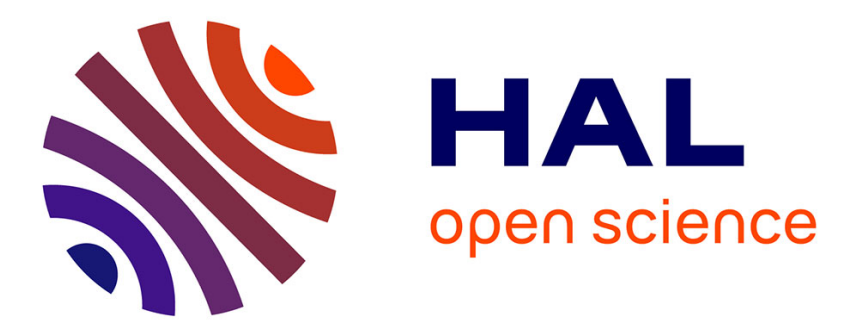

\title{
Resistive high-impedance surfaces (RHIS) as absorbers for oblique incidence electromagnetic waves
}

\author{
Yenni Pinto, Julien Sarrazin, Anne Claire Lepage, Xavier Begaud, Nicolas
}

Capet

\section{- To cite this version:}

Yenni Pinto, Julien Sarrazin, Anne Claire Lepage, Xavier Begaud, Nicolas Capet. Resistive highimpedance surfaces (RHIS) as absorbers for oblique incidence electromagnetic waves. Applied physics. A, Materials science \& processing, 2014, 117 (2), pp.693 - 697. 10.1007/s00339-014-8724-5 . hal01060603

\section{HAL Id: hal-01060603 \\ https://hal.sorbonne-universite.fr/hal-01060603}

Submitted on 10 Sep 2019

HAL is a multi-disciplinary open access archive for the deposit and dissemination of scientific research documents, whether they are published or not. The documents may come from teaching and research institutions in France or abroad, or from public or private research centers.
L'archive ouverte pluridisciplinaire HAL, est destinée au dépôt et à la diffusion de documents scientifiques de niveau recherche, publiés ou non, émanant des établissements d'enseignement et de recherche français ou étrangers, des laboratoires publics ou privés. 


\title{
Resistive high-impedance surfaces (RHIS) as absorbers for oblique incidence electromagnetic waves
}

\author{
Yenny Pinto $\cdot$ Julien Sarrazin $\cdot$ Anne-Claire Lepage • \\ Xavier Begaud $\cdot$ Nicolas Capet
}

\begin{abstract}
This paper presents a lightweight microwave absorber suitable for space applications. The absorber is based on a resistive high-impedance surface (RHIS) optimized to achieve reflection under $-15 \mathrm{~dB}$ in the band $(2-2.3 \mathrm{GHz})$ at oblique incidence for transverse electric (TE) and transverse magnetic (TM) polarizations. A first classical isotropic RHIS structure is shown to be limited to angles of incidence up to $40^{\circ}$ for TE polarized waves and up to $35^{\circ}$ for TM polarized waves. So the objective of this contribution is to present a second solution based on an anisotropic RHIS structure which presents good absorption of incident waves in TE and TM polarizations for larger angles of incidence. An example is presented for an incidence angle of $65^{\circ}$.
\end{abstract}

\section{Introduction}

Microwave absorbers can drastically decrease the reflection of incident electromagnetic waves within a certain

\author{
Y. Pinto $\cdot$ A.-C. Lepage $\cdot$ X. Begaud $(\bowtie)$ \\ Institut Mines Télécom, Télécom ParisTech - CNRS LTCI, \\ Paris, France \\ e-mail: xavier.begaud@telecom-paristech.fr \\ Y. Pinto \\ e-mail: yenny-constanza.pinto-ballesteros@ telecom-paristech.fr \\ A.-C. Lepage \\ e-mail: Anne-Claire.Lepage@telecom-paristech.fr \\ J. Sarrazin \\ Sorbonne Universités, UPMC Univ Paris 06, UR2, L2E, \\ 75005 Paris, France \\ e-mail: julien.sarrazin@upmc.fr \\ N. Capet \\ Centre National d'Etudes Spatiales, Toulouse, France \\ e-mail: nicolas.capet@cnes.fr
}

frequency range. This property may be used to solve some electromagnetic compatibility problems. For space applications, the potential of absorbers covering satellite's sides in order to reduce antenna interferences and thus improving performances of some radar systems has been investigated. To do so, ultra-light absorbers able to handle extreme space conditions (wide range of temperatures, resistance to corrosion and UV...) are required.

One popular approach to design an absorber is the Salisbury screen [1]. It consists of a resistive sheet located above a ground plane at a distance of a quarter wavelength. This structure is simple but has an inherently narrow band. To increase the operational bandwidth, Jaumann [2] absorbers use several stacked dielectric layers with resistive sheets at their interface. However, this technique leads to a thick and heavy structure which is not appropriate for being embedded on satellites, mainly because of its weight.

Recently, artificial impedance surfaces (AIS) or highimpedance surfaces (HIS) have been used as absorbers. An HIS consists of a frequency selective surface (FSS) over a grounded dielectric slab. The FSS is usually a periodic array of printed patterns loaded with resistors or resistive sheets in order to achieve absorption. The structure is known as a resistive high-impedance surface (RHIS) [3-6]. RHIS are usually thinner since no quarter wavelength resonances are involved. Consequently, RHIS appear to be a good candidate to achieve a low profile and light absorber, mandatory for space applications. However, two major limitations exist. Being a resonant structure, RHIS are intrinsically narrow band. This can be overcome by increasing the distance between the FSS and the ground plane [7], of course at the expense of the thickness. The second limitation is that absorption performance depends on the incoming wave's angle of incidence. Usually, absorber designs are optimized for incident waves at normal incidence. Some research 
groups developed some oblique optimizations for Jaumann and Circuit Analog absorbers [8-10] or other thick structures $[11,12]$, but there are relatively few works regarding the oblique incidence issue for HIS-based absorbers.

Among these few studies, some of them investigate a way to make absorbers insensitive to the angular dispersion. For example in [13], the authors use an analytical model to clearly show that increasing the permittivity of the substrate separating the ground plane from the FSS decreases the incidence angle sensitivity. This approach can be applied when the space between the FSS and the ground plane is fully (or mostly) filled by dielectric. However, achieving a light absorber involves the use of light spacer structures such as honeycomb or foam, commonly found in the space field. These materials have intrinsically a low permittivity (typically less than 1.2) and are therefore not suitable for this approach. Another way is to use ultra-thin RHIS. In fact, a short distance between the FSS and the ground plane decreases the angular dispersion. For example, in [14], an absorption defined by a reflection coefficient less than $-10 \mathrm{~dB}$ is achieved for angles ranging up to $45^{\circ}$, both in TE and TM cases. However, the extremely low profile of the structure $\left(0.017 \lambda_{0}\right)$ leads to a narrow bandwidth $(2.7 \%)$. For the targeted application, absorption bandwidths of about $14 \%$ are expected, so this technique is not suitable.

So to the authors' knowledge, no wideband and light HIS-based structure capable of an absorption at large incident angles (greater than 50-60 $)$ for simultaneously TE and TM polarizations has been reported in the literature so far. Since achieving lightweight absorbers insensitive to angle dispersion appears to be a difficult task, we therefore propose a different approach to overcome this problem. In some particular applications, the angle of incidence of the incident interfering wave can be known. This is the case in some radar systems embedded on satellites, for example. Thus, in respect to a given system, optimizing the absorber for a particular oblique incidence rather than achieving an angle insensitive absorber is an acceptable option.

Consequently, in this paper, we demonstrate the feasibility of a lightweight microwave absorber suitable for space applications optimized for oblique incidence. First of all, Sect. 2 describes the design of a light RHIS classically optimized for normal incidence and the absorber's limitations in terms of angular dispersion are highlighted. Section 3 presents the proposed solution. It consists of an anisotropic RHIS optimized simultaneously for TE and TM oblique polarizations. Finally, a conclusion is drawn in Sect. 4.

\section{Classical RHIS design}

A typical RHIS consists of an array of square patches interconnected by resistors above a grounded dielectric slab. The analytical model of this structure is explained in
[3]. The equivalent RHIS circuit consists of a RLC parallel circuit, where the surface impedance $\left(Z_{\mathrm{s}}\right)$ is calculated as indicated in (1) and (2). The real part of $Z_{\mathrm{s}}$ corresponds to the resistance $(R)$ and the imaginary part is set by the patch dimensions (capacitive response $Z_{\mathrm{C}}$ ) and metal-backed substrate (inductive response $Z_{\mathrm{L}}$ ). Circuit losses are mainly induced by the resistors, the resonance conditions are reached when the imaginary part is zero.

$Z_{\mathrm{S}}^{-1}=R^{-1}+Z_{\mathrm{L}}^{-1}+Z_{\mathrm{C}}^{-1}$

$Z_{\mathrm{S}}=\frac{R}{1-j R\left(\frac{1-\omega^{2} L C}{L \omega}\right)}$

In order to calculate the absorption, this circuit is considered as the load of a transmission line model with free space characteristic impedance $\left(Z_{0}\right)$. The reflection coefficients $\Gamma^{\mathrm{TE}}$ and $\Gamma^{\mathrm{TM}}$ for $\mathrm{TE}$ and $\mathrm{TM}$ polarizations, respectively, are obtained as indicated in (3) and (4). $Z_{\mathrm{s}}^{\mathrm{TE}}$ and $Z_{\mathrm{s}}^{\mathrm{TM}}$ are the surface impedance for TE and TM polarizations, and $\theta$ is the angle of incidence.

$\Gamma^{\mathrm{TE}}=\frac{Z_{\mathrm{s}}^{\mathrm{TE}} \cos \theta-Z_{0}}{Z_{\mathrm{s}}^{\mathrm{TE}} \cos \theta+Z_{0}}$

$\Gamma^{\mathrm{TM}}=\frac{Z_{\mathrm{s}}^{\mathrm{TM}}-Z_{0} \cdot \cos \theta}{Z_{\mathrm{s}}^{\mathrm{TM}}+Z_{0} \cdot \cos \theta}$

The maximal absorption is obtained at the resonance, when the reflection coefficient approaches to zero, in that case $Z_{\mathrm{s}}$ equals to (5) or (6) for TE and TM polarizations, respectively. Thus, the imaginary part of $Z_{\mathrm{s}}$ is equal to zero, and the impedance value is equal to the real part that corresponds to the resistance $(R)$.

$Z_{\mathrm{S}}^{\mathrm{TE}}=\frac{Z_{0}}{\cos \theta}$

$Z_{\mathrm{s}}^{\mathrm{TM}}=Z_{0} \cdot \cos \theta$

In Fig. 1, it appears that the surface impedance at the resonance depends on the angle of incidence for two

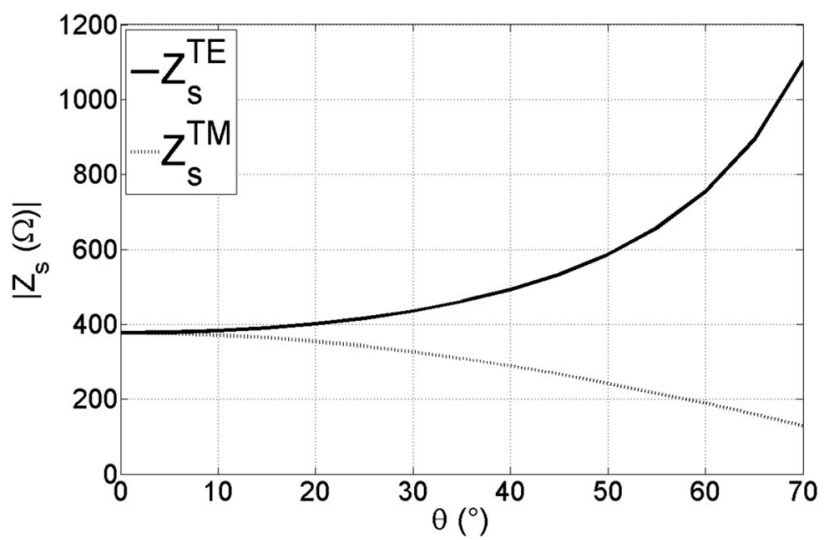

Fig. 1 RHIS surface impedance at the resonance for two polarizations (TE and TM) as a function of incidence angle 
polarizations (TE and TM). In the case of TE polarization, the optimal resistance value for maximal absorption increases as the incidence angle rises, whereas for TM polarization, the optimal resistance decreases as the incidence angle increases. So it appears that with isotropic structure, a straightforward simultaneous optimization for both TE and TM polarizations is not possible.

In [15], we proposed a lightweight solution to fabricate wideband RHIS for normal incidence. It consists of an array of square patches etched on Rogers ${ }^{\circledR} \mathrm{RO} 4003$ substrate $\left(\varepsilon_{\mathrm{r}}=3.38 \pm 0.05\right)$ on the top of a honeycomb layer $\left(\varepsilon_{\mathrm{r}}=1.08 \pm 0.05\right)$ with the ground plane (copper film) at the bottom. The resistors are implemented in a TICER ${ }^{\circledR}$ sheet with resistivity $R_{\mathrm{S}}=100 \Omega /$ square and thickness $t=0.1 \mu \mathrm{m}$. Figure 2 shows the unit cell of the RHIS structure. This structure, optimized at normal incidence $\left(\theta=0^{\circ}\right)$, presents a reflection coefficient magnitude lower than $-15 \mathrm{~dB}$ from 2 to $2.3 \mathrm{GHz}$, for TE and TM polarizations. Simulation and measurement results showed that the RHIS performs well in the required band, at normal incidence, for both TE and TM polarizations. But when the angle of incidence increases, the absorption level decreases and the frequency band shifts toward higher frequencies. Therefore, the absorption in the $2-2.3 \mathrm{GHz}$ band is limited to incidence angles up to $40^{\circ}$ for waves in TE polarization and $35^{\circ}$ for waves in $\mathrm{TM}$ polarization.

\section{Anisotropic RHIS}

In this section, an optimization strategy based on a hybrid structure is proposed. The design consists in the

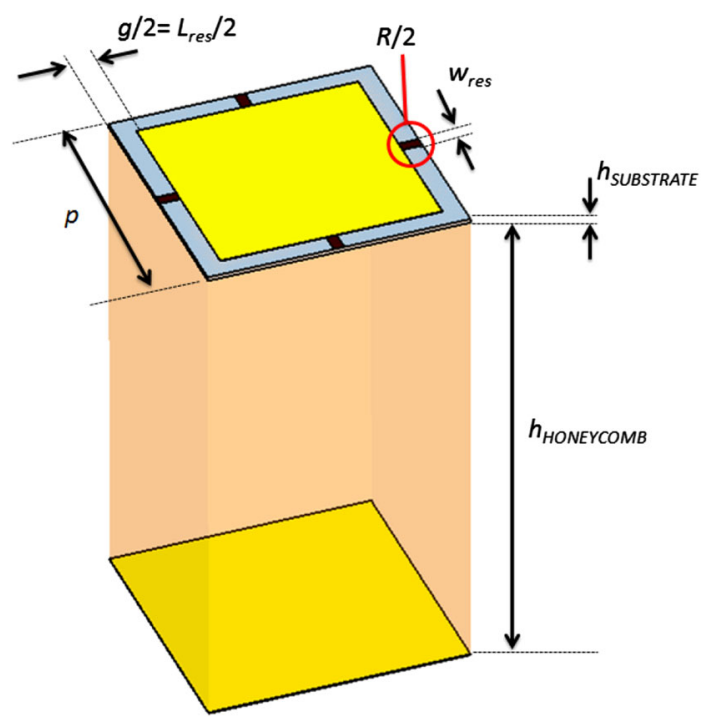

Fig. 2 RHIS unit cell $\left(h_{\text {HONEYCOMB }}=25 \mathrm{~mm}, h_{\text {SUBSTRATE }}=\right.$ $0.203 \mathrm{~mm}, p=13 \mathrm{~mm}, g=2 \mathrm{~mm}, W_{\text {res }}=0.53 \mathrm{~mm}, L_{\text {res }}=2 \mathrm{~mm}$, $R=377 \Omega$ ) combination of two classical RHIS structures, where one dimension is optimized for TE polarization and the other one for TM polarization (Fig. 3), leading then to anisotropic RHIS.

Thus, the structure is formed by rectangular patches instead of square patches and the patch's interconnection is made using different resistance values on each rectangle side. The RHIS anisotropic unit cell is shown in Fig. 4. Dimension $p_{\mathrm{TM}}\left(p_{\mathrm{TM}}=g+l_{\mathrm{TM}}\right)$ and resistance $R_{\mathrm{TM}}$ are optimized for $\mathrm{TM}$ polarization and $p_{\mathrm{TE}}\left(p_{\mathrm{TE}}=g+l_{\mathrm{TE}}\right)$ and $R_{\mathrm{TE}}$ for TE polarization.

This RHIS is then optimized to achieve a reflection lower than $-15 \mathrm{~dB}$ in the band $(2-2.3 \mathrm{GHz})$ for TE and $\mathrm{TM}$ polarizations at $65^{\circ}$ angle of incidence. The wave vector $k$ is supposed to lie in the $X Z$-plane. The structure is made of the same materials with same characteristics as the classical isotropic RHIS presented in the previous section.

The RHIS anisotropic unit cell is simulated using CST Microwave Studio $\subset$. The simulated results are plotted in

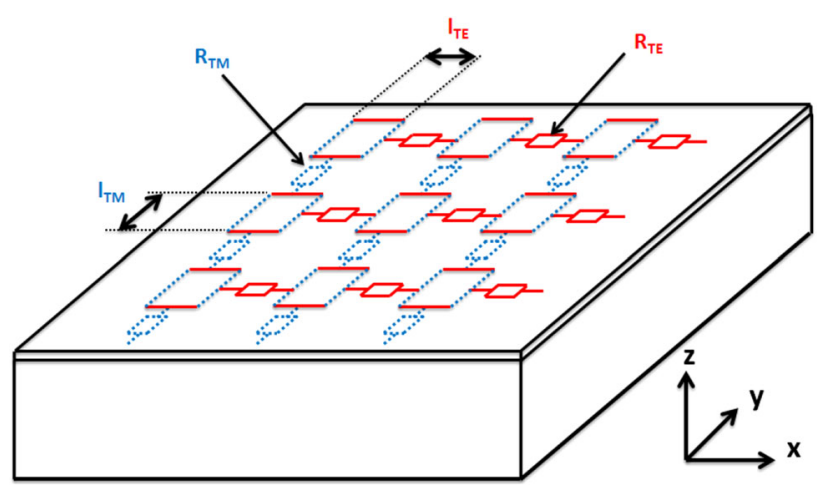

Fig. 3 Configuration of the RHIS with anisotropic geometry

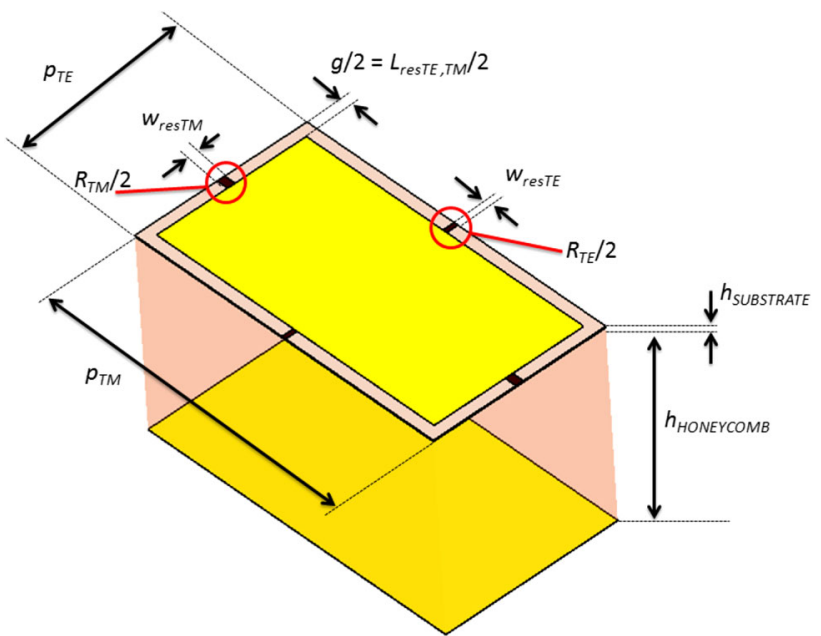

Fig. 4 Anisotropic RHIS unit cell $\left(h_{\text {HONEYCOMB }}=25 \mathrm{~mm}, h_{\text {SUB- }}\right.$ STRATE $=0.203 \mathrm{~mm}, p_{\mathrm{TE}}=22.8 \mathrm{~mm}, p_{\mathrm{TM}}=42.5 \mathrm{~mm}, g=3 \mathrm{~mm}$, $W_{\text {resTE }}=0.55 \mathrm{~mm}, \quad W_{\text {resTM }}=1 \mathrm{~mm}, \quad R_{\mathrm{TE}}=444 \Omega \quad$ and $R_{\mathrm{TM}}=333 \Omega$ ) 


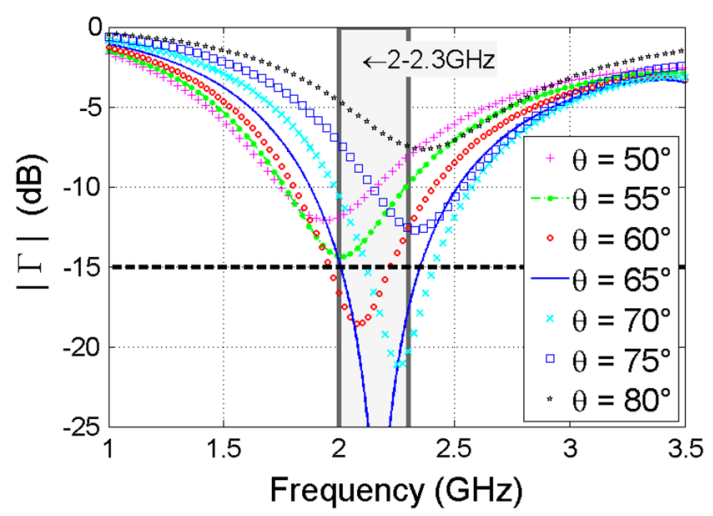

Fig. 5 Reflection coefficient for different angles of incidence for TE polarization

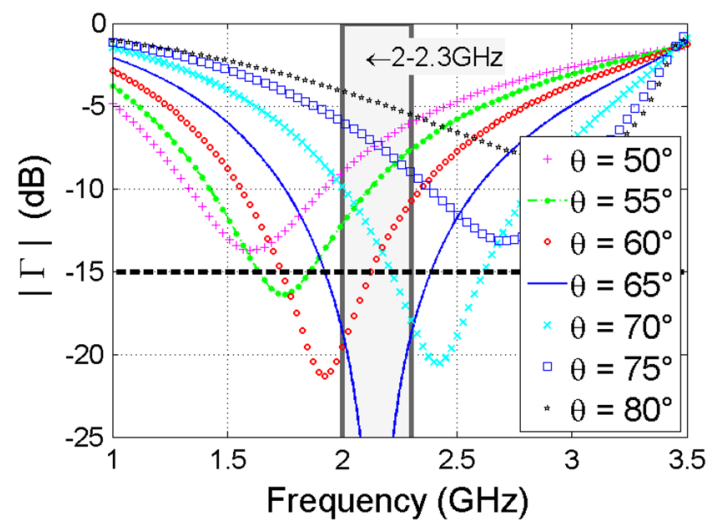

Fig. 6 Reflection coefficient for different angles of incidence for TM polarization

Figs. 5 and 6; the required performance is obtained for $65^{\circ}$ angle of incidence in both TE and TM polarizations.

These results show that absorbing incident waves in a particular oblique incidence for both TE and TM polarizations simultaneously is feasible using an RHIS structure with anisotropic geometry. However, the angular dispersion is restricted to $\pm 5^{\circ}$ around the optimal angle of incidence, with a $-15 \mathrm{~dB}$ reflection criteria in both TE and TM polarizations, but the frequency band is shifted. The frequency shift is more significant in TM polarization.

In order to validate the concept, simulation results have been compared to measurements. The anisotropic RHIS prototype is realized using the same materials and method as those of the isotropic RHIS prototype described in [15]. The substrate slab with the patches and resistors is pasted on the top of honeycomb using an adhesive film. And a copper film is pasted at the honeycomb bottom. Figure 7 shows the prototype.

The measurement of the reflection coefficient is carried out as detailed in [15]. The reflection coefficient is

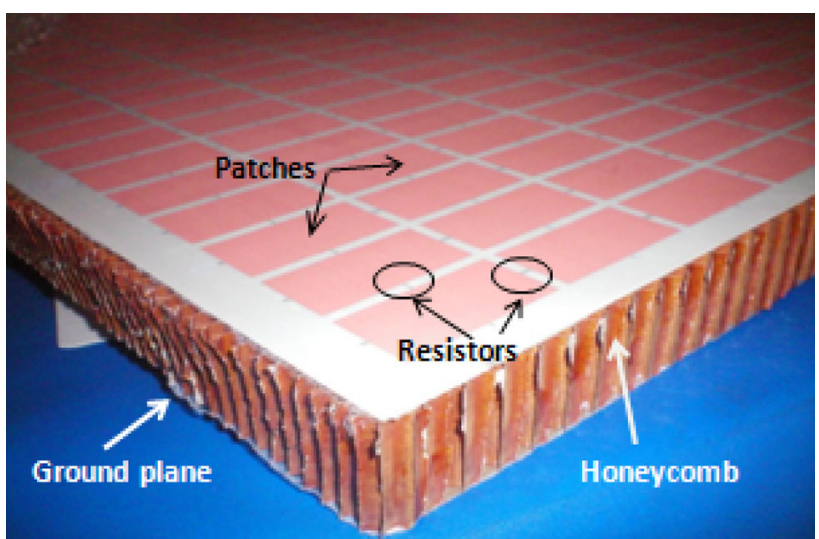

Fig. 7 Anisotropic RHIS prototype

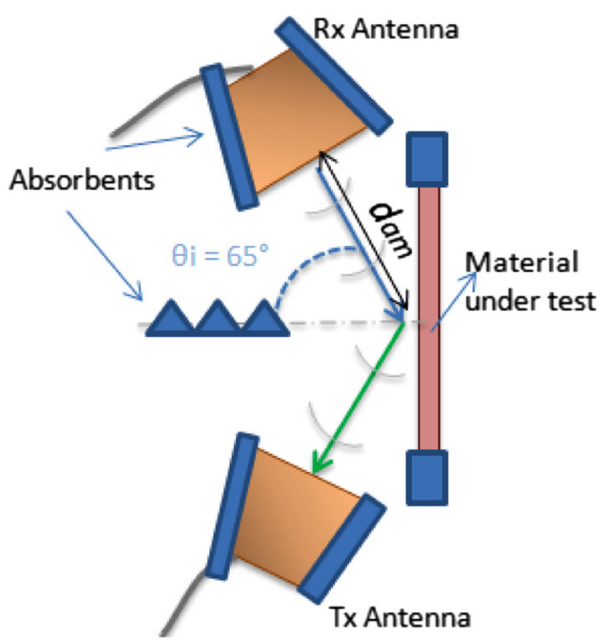

Fig. 8 Measurement setup at oblique incidence

measured using two horn antennas. The two horn antennas are connected to a network analyzer E5071C Agilent. The Fig. 8 illustrates the measurement setup at oblique incidence. Measurements are taken in an anechoic chamber, RHIS anisotropic edges and antennas are cover with absorbents in order to reduce environment interferences. The distance between the antenna and anisotropic RHIS prototype is $d_{\mathrm{am}}=107 \mathrm{~cm}$. The anisotropic RHIS prototype is fixed and the antennas are placed according to incidence angle $\left(\theta_{\mathrm{i}}=\theta_{\mathrm{r}}\right)$.

The measurements are taken for oblique incidence $\left(65^{\circ}\right)$. The results are plotted in Fig. 9 for TE polarization and measurements for TM polarization are presented in Fig. 10.

A good agreement between results is obtained, however, differences in frequency and level are observed.

Shifts in frequency and level can be explained by different ways: 


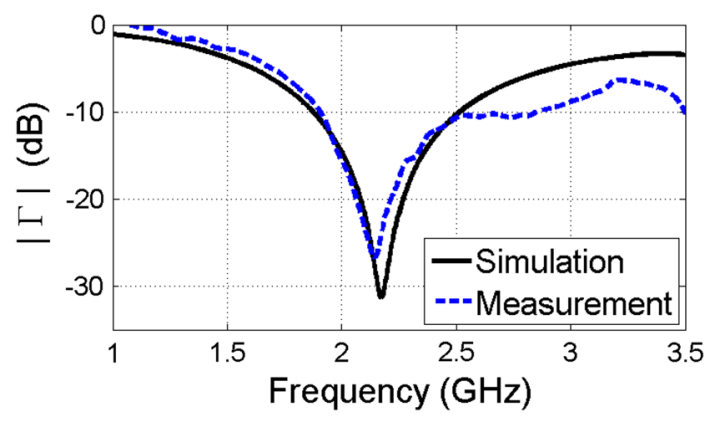

Fig. 9 Comparison between simulation and measurement results at oblique incidence $\left(65^{\circ}\right)$. TE polarization

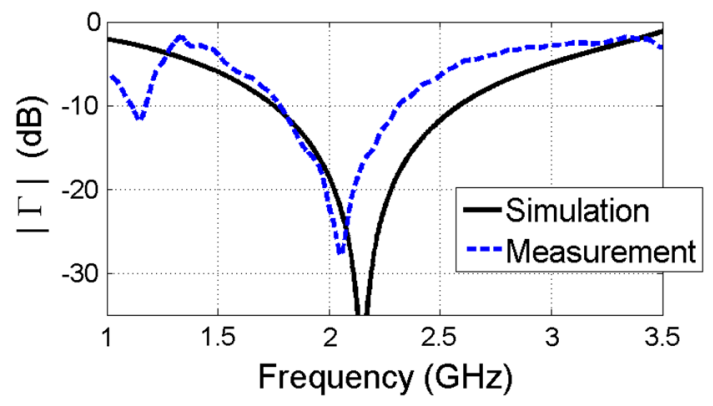

Fig. 10 Comparison between simulation and measurement results at oblique incidence $\left(65^{\circ}\right)$. TM polarization

- The imperfections of realization.

- The simulation does not take into account the presence of the double-sided adhesive film.

- The resistance value is not accurate and has a tolerance of $+/-10 \%$.

- The permittivity value of honeycomb is not accurate.

\section{Conclusions}

The design of a lightweight absorbing material for larger angles of incidence based on a RHIS has been described. It has been firstly highlighted that the classic isotropic RHIS can meet the required specifications at normal incidence but only up to a $40^{\circ}$ incidence for waves in TE polarization and $35^{\circ}$ for waves in TM polarization. Hence a second solution based on an anisotropic RHIS structure has been proposed. This solution performs well for a given angle of incidence in both TE and TM polarizations. The final design has been optimized to absorb $65^{\circ}$ incident waves on both TE and TM polarizations simultaneously. Simulations have been validated by measurements.

\section{References}

1. W.W. Salisbury, Absorbent, Body for Electromagnetic Waves, U.S. Patent 2599944, June 1952

2. L.J. Du Toit, The design of Jaumann absorbers. IEEE Antennas Propag. Mag. 36(6), 17-25 (1994)

3. F. Linot, X. Begaud, M. Soiron, C. Renard, M. Labeyrie, Characterization of a loaded high impedance surface. Int. J. Microw. Wirel. Technol. 1, 483-487 (2009)

4. F. Costa, A. Monorchio, G. Manara, Analysis and design of ultra thin electromagnetic absorbers comprising resistively loaded high impedance surfaces. IEEE Trans. Antennas Propag. 58(5), 1551-1558 (2010)

5. Y. Tsuda, T. Yasuzumi, O. Hashimoto, A thinwave absorber using closely placed divided conductive film and resistive film. IEEE Antennas Wirel. Propag. Lett. 10, 892-895 (2011)

6. M. Li, S.Q. Xiao, Y.Y. Bai, B.Z. Wang, An ultrathin and broadband radar absorber using resistive FSS. IEEE Antennas Wirel. Propag. Lett. 11, 748-751 (2012)

7. Y. Pang, H. Cheng, Y. Zhou, J. Wang, Upper bound for the bandwidth of ultrathin absorbers comprising high impedance surfaces. IEEE Antennas Wirel. Propag. Lett. 11, 224-227 (2012)

8. B.A. Munk, P. Munk, J. Pryor, On designing Jaumann and circuit analog absorbers (CA absorbers) for oblique angle of incidence. IEEE Trans. Antennas Propag. 55(1), 186-193 (2007)

9. A. Kazemzadeh, A. Karlsson, Multilayered wideband absorbers for oblique angle of incidence. IEEE Trans. Antennas Propag. 58(11), 3637-3646 (2010)

10. B. Chambers, A. Tennant, Design of wideband Jaumann radar absorbers with optimum oblique incidence performance. Electron. Lett. 30(18), 1530-1532 (1994)

11. R. Janaswamy, Oblique scattering from lossy periodic surfaces with application to anechoic chamber absorbers. IEEE Trans. Antennas Propag. 40(2), 162-169 (1992)

12. H. Cho, H. Ling, C.H. Liang, Design of corrugated absorbers for oblique incidence using genetic algorithm. IEEE Antennas and Propagation Society International Symposium 4, 708-711 (2001)

13. O. Luukkonen, F. Costa, C.R. Simovski, A. Monorchio, S.A. Tretyakov, A thin electromagnetic absorber for wide incidence angles and both polarizations. IEEE Trans. Antennas Propag. 57(10), 3119-3125 (2009)

14. B.Q. Lin, C.M. Tong, W. Li, H.B. Niu, H. Zhou, An ultrathin electromagnetic absorber comprising resistive resonance loops. IEEE Antennas Wirel. Propag. Lett. 11, 1021-1023 (2012)

15. Y. Pinto, J. Sarrazin, A.C. Lepage, X. Begaud, N. Capet, Design and measurement of a thin and light absorbing material for space applications. Appl. Phys. A 115(2), 541-545 (2014) 